Journal on Satisfiability, Boolean Modeling and Computation 5 (2008) 217-242

Research Note

\title{
A Resolution Based SAT-solver Operating on Complete Assignments
}

\author{
Eugene Goldberg \\ Cadence Research Labs, \\ 2150 Shattuck Ave., 10th floor, Berkeley, \\ California, 94704, USA
}

egold@cadence.com

\begin{abstract}
Most successful systematic SAT-solvers are descendants of the DPLL procedure and so operate on partial assignments. Using partial assignments is explained by the "enumerative semantics" of the DPLL procedure. Current clause learning SAT-solvers, in a sense, have outgrown this semantics. Instead of enumerating the search space as the DPLL procedure does, they explicitly build a resolution proof. In this paper, we suggest a semantics that, in our opinion, is more suitable for clause learning SAT-solvers. The idea is to consider a set of complete assignments not just as a part of the search space but as an "encryption" of a resolution proof or a part thereof. Importantly, a set of points encrypting a resolution proof can be dramatically smaller than the entire search space. We introduce a resolution based SAT-solver with clause learning called FI (short for Find point Image of a proof) that is inspired by the new semantics. FI operates on complete assignments. We compare our naive implementation of FI with Minisat and BerkMin. Experiments show that FI is competitive with Minisat and BerkMin in terms of backtracks. In terms of performance, FI is slower than Minisat and BerkMin for small CNF formulas. On the other hand, even the current primitive implementation of FI is competitive with Minisat and BerkMin on large Bounded Model Checking formulas due to its superior decision making.
\end{abstract}

KEYWORDS: SAT-solver, resolution, decision-making, local search, complete assignment

Submitted March 2007; revised April 2008; published June 2008

\section{Introduction}

The resolution proof system [1] has achieved outstanding popularity in practical applications. The best systematic SAT-solvers are descendants of the DPLL procedure [3] that can be simulated by so-called tree-like resolution (a special type of general resolution). The DPLL procedure operates on partial assignments. (An assignment is called partial if some of the formula variables are not assigned any value). The current partial assignment is extended until a clause is falsified. Then, the DPLL procedure backtracks to the last decision assignment and flips it.

The reason for using partial rather than complete assignments is that by rejecting a partial assignment the DPLL procedure may simultaneously reject an exponential number of complete assignments. (An assignment is called complete if every variable of the formula is assigned a value.) This greedy approach is a natural consequence of enumerative 
semantics of DPLL. The DPLL procedure partitions the search space into non-overlapping subspaces corresponding to the leaves of the search tree. For this reason, one can view this procedure as enumeration of the complete assignments (or points) of the search space in a particular order. (From now on we will use the terms complete assignments and points interchangeably.) So, by enumerative semantics we mean the interpretation of DPLL as a method for enumerating the points of the search space.

In this paper, we are making a case for using complete assignments in resolution based SAT-solvers. This case is based on the following observations and results.

- Current resolution based SAT-solvers use clause learning. So instead of explicitly enumerating the search space as it is done in the DPLL procedure, they actually build a resolution proof. These SAT-solvers have outgrown the enumerative semantics of DPLL.

- Using complete assignments is justified by the proof encryption semantics we introduce in this paper. The proof encryption semantics can be viewed as a replacement of enumerative semantics for SAT-solvers with clause learning. In this semantics, a set of complete assignments is considered as an encryption of a resolution proof or a part thereof. Proof encryption semantics gives a more precise metric for measuring the quality of a set of points examined by a SAT-solver. Proof encryption semantics implies that it is more important for a SAT-solver to try to visit a particular set of points rather than attempt to enumerate all the points of the search space as fast as possible.

- We introduce a resolution based SAT-solver FI (Find point Image of a proof) with clause learning that operates on complete assignments. The set of points visited by $F I$ forms an encryption of the proof FI builds. So FI can be viewed as a SAT-solver that builds a proof encryption along with the proof. Experimental results show that for large industrial formulas, even a very primitive implementation of $F I$ is competitive with state-of-the-art SAT-solvers.

- FI can be viewed as a regular SAT-solver with clause learning whose choice of branching variables is limited to the variables of clauses falsified by the current complete assignment. Importantly, experiments show that good performance of $F I$ can not be explained by great robustness of decision making based on computing conflict activity of literals/variables that was first introduced in [14]. So FI performance looks mysterious in the enumerative semantics. On the other hand, it has a natural explanation in proof encryption semantics.

- Complete assignments have more information about the formula than partial ones. For example, when a CNF formula $F$ has a small unsatisfiable core $F^{\prime}$, any complete assignment $\boldsymbol{p}$ falsifies at least one clause of $F^{\prime}$. If one restricts the choice of branching variables to those of clauses falsified by $\boldsymbol{p}$, then there is a high probability to pick a variable of $F^{\prime}$ as a branching variable. On the other hand, a SAT-solver operating on partial assignments may get stuck in the clauses of $F \backslash F^{\prime}$ never reaching clauses of $F^{\prime}$. The reason is that it has to choose the next branching variable out of all free variables of $F$, which significantly reduces the probability of picking a variable of $F^{\prime}$. 
- Greater informational capacity of complete assignments can be also leveraged when solving satisfiable formulas. When backtracking, the complete assignment maintained by $F I$ remembers last assignments made in the subspace FI leaves. (A SAT-solver operating on partial assignments stores this information only implicitly through conflict clauses.) Besides, FI reports satisfiability as soon as the set of clauses falsified by the current complete assignment is empty. This may happen long before all clauses are satisfied by fixed assignments. On the contrary, a SAT-solver operating on partial assignments reports satisfiability only when all clauses of the formula are satisfied by fixed assignments.

Although, resolution based SAT-solvers like Grasp [19],SATO [20], Chaff [14],BerkMin [8], Siege and Minisat [4] are considered as descendants of DPLL, they are different from DPLL in one very important aspect. Namely, they use clause learning (first introduced in Grasp). For an unsatisfiable formula $F$, the DPLL procedure terminates if the right branch of the root node of the search tree is proved not to contain a satisfying assignment. So the DPLL procedure has to maintain a binary search tree. On the other hand, a clause learning SAT-solver terminates when an empty clause is derived. For that reason, clause learning SAT-solvers enjoy great flexibility in organizing search e.g. they can perform frequent restarts. (In addition to greater flexibility, adding learned clauses makes a SAT-solver more powerful [2].) One can say that clause learning procedures only implicitly enumerate the search space, while building a resolution proof is what these SAT-solvers do explicitly. (Even if a clause learning SAT-solver does not generate a resolution proof, such a proof can be extracted from the set of conflict clauses [9].) In a sense, current resolution based SATsolvers have outgrown the enumerative semantics of DPLL. New decision making heuristics introduced by Chaff and then improved by BerkMin, Siege, Minisat and others can be viewed as the recognition of the fact that clause learning SAT-solvers build a resolution proof. Computation of literal/variable activity based on learned conflict clauses is nothing else but decision making driven by a resolution proof.

The idea of proof encryption semantics is based on the following observation Let $F$ be a CNF formula and $T$ be a set of complete assignments to the variables of $F$. Let $S A T(T, F)$ be a procedure that resolves only the clauses of $F$ and their resolvents that are specified by the points of $T$ (this procedure is described in Section 3 in more detail) Suppose $\operatorname{SAT}(T, F)$ results in derivation of an empty clause, which means that $S A T(T, F)$ generated a resolution proof $R$ that $F$ is unsatisfiable. We will say that $T$ is a point image of $R$. One can view a point image of $R$ as its encryption in terms of complete assignments. Importantly, as we show in this paper, every resolution proof $R$ that a CNF formula $F$ is unsatisfiable has a point image $T$ whose size is at most twice the size of $R$ (in terms of resolution operations). One can view the interpretation of a set of points as an encryption of a resolution proof as an alternative to the enumerative semantics. (We will refer to this alternative semantics as proof encryption semantics). The fact that the size of a point image of a resolution proof can be dramatically smaller than the size of the entire search space, questions the applicability of enumerative semantics to generation of resolution proofs. The notion of a point image implies that it is much more important to visit a particular set of points (which comprises a very small subset of the entire search space) rather than enumerate all the points as fast as possible. 
A straightforward way to use the notion of a point image of a proof is as follows. First, a set $T$ of points that is a point image of a resolution proof (or at least has a high chance to be such) is generated. Second, the $S A T(T, F)$ procedure above is applied to generate a resolution proof. This approach has at least two problems. Firstly, it is not clear how to generate a good set of points $T$. Second, even if we find a way to generate $T$, the $S A T(T, F)$ procedure mentioned above is not practical because it may generate a very large number of redundant resolvents. (The reason for introducing $S A T(T, F)$ is to formalize the notion of proof encryption.)

In this paper, we describe a SAT-solver FI inspired by the proof encryption semantics. The main idea of $F I$ is to use complete assignments to control decision making of a DPLLlike procedure. That is instead of constructing a set of points $T$ and a proof $R$ separately, FI builds them together. As we show later, the set of points visited by FI (after a natural extension) indeed contains a point image of the resolution proof FI builds.

Depending on semantics used, one can give two interpretations of $F I$ that are equivalent algorithmically. In the first interpretation, FI is considered as a DPLL-like SAT-solver with clause learning that is allowed to make assignments only to variables of clauses that are falsified by a complete assignment $\boldsymbol{p}$. This complete assignment dynamically changes every time $F I$ assigns a variable $x_{i}$ a value that disagrees with the value of $x_{i}$ in $\boldsymbol{p}$.

In the second interpretation, FI operates on complete assignments. In this interpretation, all variables are assigned and so there are no free variables. However, a value of variable $x_{i}$ can be fixed so that all the points visited by $F I$ after that have the same value of $x_{i}$ until the latter gets unfixed.

The first interpretation is easier to understand from the operational point of view. In this interpretation, FI is just a "regular" clause learning SAT-solver in which the choice of variables to branch on is limited. The second interpretation better explains the semantics of $F I$ which is visiting a set of points encrypting a resolution proof.

In the experiments described in this paper, we used a very simple implementation of $F I$ that lacked many techniques employed by state-of-the art SAT-solvers (like fast BCP, efficient data structures, special treatment of binary clauses, removal of inactive conflict clauses and so on). We compared FI with SAT-solvers BerkMin and Minisat. The experiments showed that while FI was competitive with BerkMin and Minisat in terms of backtracks, it was slower on small and medium size formulas. However, when we run these SAT-solvers on large BMC formulas (up to a few millions of variables) the performance of FI was comparable with that of BerkMin and Minisat. In this case, the superior decision making of FI compensated for the inefficiency of implementation. Note that the good performance of $F I$ can not be explained by enumerative semantics. In particular, we show that the SAT-solver different from $F I$ only in that it picks a window of variables randomly (rather than uses the window specified by the set of clauses falsified by $\boldsymbol{p}$ ) works much worse.

This paper is structured as follows. Section 2 describes FI. Section 3 introduces proof encryption semantics. In Section 4, we argue that complete assignments contain more information about the formula than partial ones. Section 5 compares FI with local search and DPLL-based procedures. Experimental results are given in Section 6. We make some conclusions in Section 7. 


\section{Description of $\boldsymbol{F I}$}

In this section, we describe the SAT-solver FI. As mentioned in the introduction, there are two interpretations of $F I$. This section uses the first interpretation i.e. we consider $F I$ as a regular SAT-solver with clause learning whose decision making is driven by a complete assignment $\boldsymbol{p}$. We will call assignments made by $F I$ to decision and implied variables fixed to distinguish them from those assignments of $\boldsymbol{p}$ that still can be changed.

This section is structured as follows. In Subsection 2.1, we recall some basic definitions. Subsection 2.2 gives an example of how FI works. Subsection 2.3 gives the pseudo-code of the main procedure of FI. The decision making of $F I$ is explained in Subsection 2.4. The other features of $F I$ (BCP, conflict clause generation, restarts and initial point generation) are described in Subsections 2.5, 2.6. Some details of the implementation of FI we used in experiments will be described in Subsection 6.1.

\subsection{Basic definitions}

Let $F$ be a CNF formula (i.e. conjunction of disjunctions of literals) over a set $X$ of Boolean variables. The satisfiability problem (SAT) is to find a complete assignment $\boldsymbol{p}$ (called a satisfying assignment) to the variables of $X$ such that $F(\boldsymbol{p})=1$ or to prove that such an assignment does not exist. If $F$ has a satisfying assignment, $F$ is called satisfiable. Otherwise, $F$ is unsatisfiable. A disjunction of literals is further referred to as a clause. A clause with one literal is called unit. A complete assignment to variables of $X$ will also be called a point of the Boolean space $B^{|X|}$ where $B=\{0,1\}$. A complete assignment $\boldsymbol{p}$ satisfies clause $C$ if $C(\boldsymbol{p})=1$. If $C(\boldsymbol{p})=0, \boldsymbol{p}$ is said to falsify $C$. We denote by $\operatorname{Vars}(\boldsymbol{C})$ and $\operatorname{Vars}(\boldsymbol{F})$ the set of variables of clause $C$ and $\mathrm{CNF}$ formula $F$ respectively.

\subsection{Example}

In this subsection, we show how $F I$ works when testing the satisfiability of the CNF formula $F$ below. Let $F$ be the CNF formula consisting of the following eight clauses $C_{1}=\overline{x_{1}} \vee x_{2}$, $C_{2}=x_{3} \vee x_{4}, C_{3}=x_{1} \vee x_{4} \vee x_{5}, C_{4}=\overline{x_{2}} \vee \overline{x_{5}}, C_{5}=\overline{x_{3}} \vee x_{5}, C_{6}=x_{2} \vee \overline{x_{4}}, C_{7}=\overline{x_{5}} \vee x_{6}$, $C_{8}=x_{1} \vee \overline{x_{3}} \vee \overline{x_{5}}$. First, $F I$ generates a complete assignment $\boldsymbol{p}$. Assume that $\boldsymbol{p}$ is equal to $\left(x_{1}=0, x_{2}=0, x_{3}=0, x_{4}=0, x_{5}=0, x_{6}=0\right)$. After generating $\boldsymbol{p}, F I$ computes the set $M(\boldsymbol{p})$ of clauses falsified by $\boldsymbol{p}$. In our case $M(\boldsymbol{p})=\left\{C_{2}, C_{3}\right\}$ because $C_{2}$ and $C_{3}$ are the only clauses of $F$ falsified by $\boldsymbol{p}$.

Then FI starts making fixed assignments to variables of $F$. However, in contrast to a regular DPLL-like SAT-solver, not all variables are available for fixed assignments. Initially, only the variables of $\operatorname{Vars}(M(\boldsymbol{p}))=\left\{x_{1}, x_{3}, x_{4}, x_{5}\right\}$ i.e. the variables of clauses $C_{2}$ and $C_{3}$ falsified by $\boldsymbol{p}$ can be used for decision making. Assume that $F I$ picks variable $x_{1}$ and makes fixed assignment $x_{1}=0$. Since $x_{1}$ is equal to 0 in $\boldsymbol{p}$, the assignment $x_{1}=0 F I$ is making agrees with $\boldsymbol{p}$ and so the latter remains the same. The set $M(\boldsymbol{p})$ does not change either. At this point, clause $C_{1}$ is satisfied (by this fixed assignment) and literal $x_{1}$ is removed from $C_{3}$ and $C_{8}$. Since no clause of $F$ becomes unit, the Boolean Constraint Propagation (BCP) procedure does not make any new fixed assignments.

Suppose that the next fixed assignment FI makes is $x_{3}=1$. (Since $x_{1}$ is already assigned a fixed value, the only variables of $\operatorname{Vars}(M(\boldsymbol{p}))$ that are available for branching are $x_{3}, x_{4}, x_{5}$. 
Since this assignment disagrees with $\boldsymbol{p}, F I$ flips the value of $x_{3}$ in $\boldsymbol{p}$, so the new current complete assignment $\boldsymbol{p}$ is $\left(x_{1}=0, x_{2}=0, x_{3}=1, x_{4}=0, x_{5}=0, x_{6}=0\right)$. Since $\boldsymbol{p}$ has changed, $M(\boldsymbol{p})$ has to be recomputed. The new value of $M(\boldsymbol{p})$ is $\left\{C_{3}, C_{5}\right\}$. After making assignment $x_{3}=1$, clause $C_{2}$ is satisfied by this fixed assignment while literal $\overline{x_{3}}$ is removed from $C_{5}$ and $C_{8}$.

Since $C_{5}$ and $C_{8}$ became unit clauses, the BCP procedure makes new fixed assignments. Assume that unit clause $C_{8}$ is processed first. To satisfy $C_{8}, F I$ makes fixed assignment $x_{5}=0$ that agrees with the current assignment $\boldsymbol{p}$ so the set $M(\boldsymbol{p})$ does not change. The fact that $x_{5}=0$ agrees with $\boldsymbol{p}$ means that the clause $C_{8}$ is satisfied by the current $\boldsymbol{p}$ and hence it is not in $M(\boldsymbol{p})$. So in contrast to a decision assignment that can be made only to a variable of $\operatorname{Vars}(M(\boldsymbol{p}))$, implied assignments performed during BCP can be made to any variable of the formula whose value has not been fixed yet. After assigning $x_{5}=0$, clause $C_{5}$ becomes unsatisfiable. (Note that at this moment $C_{5}$ is falsified by the current assignment $\boldsymbol{p}$.) $F I$ generates a conflict clause as described in Subsection 2.5. In this case, the conflict clause $C_{9}=x_{1} \vee \overline{x_{3}}$ is generated and added to $F$. Note that the conflict clause $C$ is falsified by the current point $\boldsymbol{p}$. So it has to be added to $M(\boldsymbol{p})$.

After generating a conflict clause, $F I$ backtracks to the decision assignment that is closest to the last one and is responsible for the conflict. In our case, FI backtracks to assignment $x_{1}=0$. The last decision assignment $x_{3}=1$ and all implied assignments made after $x_{3}=1$ are erased. When backtracking, the current complete assignment $\boldsymbol{p}$ does not change i.e. it remains exactly the same it was at the moment of the conflict. After backtracking, the conflict clause $C_{9}=x_{1} \vee \overline{x_{3}}$ becomes unit due to the decision assignment $x_{1}=0$ that is still fixed. So the BCP procedure is invoked. At this time $M(\boldsymbol{p})$ is equal to $\left\{C_{3}, C_{5}, C_{9}\right\}$.

The fixed assignment $x_{3}=0$ deduced from $C_{9}$, does not agree with the current complete assignment $\boldsymbol{p}=\left(x_{1}=0, x_{2}=0, x_{3}=1, x_{4}=0, x_{5}=0, x_{6}=0\right)$. So $F I$ flips the value of $x_{3}$ in $\boldsymbol{p}$ and recomputes $M(\boldsymbol{p})$. The new value of $M(\boldsymbol{p})$ is $\left\{C_{2}, C_{3}\right\}$. After assigning $x_{3}=0$, the clause $C_{2}$ becomes unit and the fixed assignment $x_{4}=1$ is deduced from it. Since $x_{4}=1$ disagrees with the current complete assignment $\boldsymbol{p}$, the value of $x_{4}$ is flipped in $\boldsymbol{p}$. So $\boldsymbol{p}$ is now equal to $\left(x_{1}=0, x_{2}=0, x_{3}=0, x_{4}=1, x_{5}=0, x_{6}=0\right)$. The new value of $M(\boldsymbol{p})$ is $\left\{C_{6}\right\}$. At this point, the clause $C_{6}$ is unit. After making the assignment $x_{2}=1$, deduced from $C_{6}$, and recomputing $\boldsymbol{p}$ and $M(\boldsymbol{p})$ we obtain $\boldsymbol{p}=\left(x_{1}=0, x_{2}=1, x_{3}=0, x_{4}=1, x_{5}=0, x_{6}=0\right)$ and $M(\boldsymbol{p})=\emptyset$. This means that $F$ is satisfiable and $\boldsymbol{p}$ is a satisfying assignment. Note that the current set of fixed assignments $\left(x_{1}=0, x_{3}=0, x_{4}=1, x_{2}=1\right)$ does not satisfy clauses $C_{4}=\overline{x_{2}} \vee \overline{x_{5}}, C_{7}=\overline{x_{5}} \vee x_{6}$ (but they are satisfied by the assignment $x_{5}=0$ of $\boldsymbol{p}$ that is not fixed.) This is an important feature of FI. It may recognize the satisfiability of a CNF formula much earlier than a SAT-solver operating on partial assignments (because such a SAT-solver has to keep making fixed assignments until each clause of the formula is satisfied by a fixed assignment).

\subsection{Main procedure}

The pseudo-code of FI (without restarts) is shown in Figure 1. An initial complete assignment is generated by the procedure generate_initial_assignment. Then the set $M(\boldsymbol{p})$ of clauses falsified by $\boldsymbol{p}$ is computed. After that, FI follows the well-known procedure of [19] used by the state-of-the-art resolution based SAT-solvers. The only difference is that FI 
maintains two additional entities: a complete assignment $\boldsymbol{p}$ and the set $M(\boldsymbol{p})$ of clauses falsified by $\boldsymbol{p}$. When $F I$ makes an assignment to a variable $x_{i}$ (either decision or implied one) it checks if the chosen value of $x_{i}$ is equal to the value of $x_{i}$ in $\boldsymbol{p}$. If these values are equal, then no re-computation of $\boldsymbol{p}$ or $M(\boldsymbol{p})$ occurs. Otherwise, a new complete assignment $\boldsymbol{p}^{\prime}$ is produced from $\boldsymbol{p}$ by flipping the value of $x_{i}$ and the set $M\left(\boldsymbol{p}^{\prime}\right)$ is computed (by recomputation of $M(\boldsymbol{p}))$. If an unsatisfiable clause is found, FI backtracks without changing the current point $\boldsymbol{p}$.

The current complete assignment $\boldsymbol{p}$ and the set $M(\boldsymbol{p})$ are used in FI solely for the purpose of decision making. Namely, the next variable to be assigned is picked only among variables of $\operatorname{Vars}(M(\boldsymbol{p}))$ i.e among the variables of the clauses currently falsified by $\boldsymbol{p}$. Note that $F I$ reports that $F$ is satisfiable as soon as the set $M(\boldsymbol{p})$ becomes empty. As we saw in the example above, this may happen before the current set of fixed assignments (decision and implied) satisfies all the clauses.

$F I$ is sound because it only adds clauses implied by the original formula $F$. FI is also complete because it never derives a conflict clause that is implied by another clause of the current formula (see below). In particular, FI never derives a conflict clause that is identical to another clause of the formula. In other words, FI adds to the formula only new clauses. So if $F$ is unsatisfiable, $F I$ will eventually derive an empty clause (because the total number of unique clauses of $n$ variables is finite and equal to $3^{n}$.) Otherwise, it will find a satisfying assignment. The reason is that every partial assignment leading to a conflict is also unique (repetition of such a partial assignment is prohibited by the corresponding conflict clause). On the other hand, the number of partial assignment is finite.

The reason why FI derives only new clauses is as follows. (The same argument applies to other SAT-solvers like Zchaff, BerkMin under the assumption that they never delete derived clauses.) FI uses complete BCP. That is at the time a new fixed decision assignment is made (after the previous BCP is over), each clause of the current formula is either satisfied by a fixed assignment or has at least two literals that are not assigned (by fixed assignments). On the other hand, a conflict clause $C$ derived by $F I$ contains exactly one literal assigned in the last BCP (i.e. BCP that has led to the conflict) and all literals of $C$ are set to 0 by fixed assignments.

Clause $C$ can not be implied by a clause $C^{\prime}$ of the current formula that was satisfied by a fixed assignment before the last BCP. The reason is that $C^{\prime}$ has to contain a literal that is not in $C$. Clause $C$ can not be implied by a clause $C^{\prime \prime}$ that was not satisfied before the last BCP. If $C^{\prime \prime}$ became satisfied in the last BCP then $C^{\prime \prime}$ has at least one literal that is not in $C$. If $C^{\prime \prime}$ became falsified by the last $\mathrm{BCP}$ it has at least two literals set to 0 in this BCP while $C$ has only one such literal. So again $C^{\prime \prime}$ has a literal that is not in $C$. Finally, if a literal of $C^{\prime \prime}$ is left unassigned this literal is not in $C$ (because all literals of $C$ are assigned).

\subsection{Decision making of FI}

The decision making of $F I$ is based on computing conflict activity of literals first introduced in Chaff [14] and then developed in BerkMin [8], Siege, Minisat [4] and other SAT-solvers. The activity of literals is computed similar to BerkMin (but in contrast to FI, BerkMin computes the activity of variables rather than literals). Let $\operatorname{lit}\left(x_{i}\right)$ be a literal of variable $x_{i}$. If $l i t\left(x_{i}\right)$ occurs in $k$ clauses of the current formula that were involved in the last conflict, 


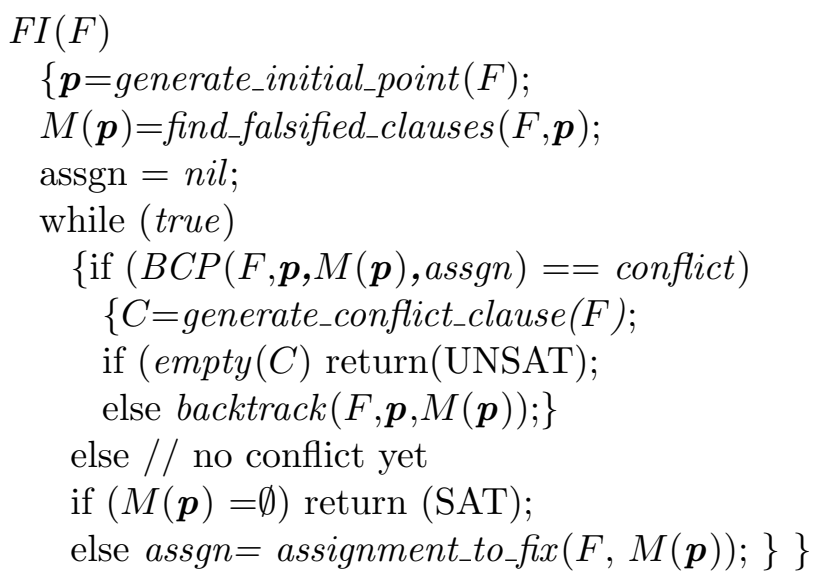

Figure 1. Pseudo-code of $F I$

then the activity of $l i t\left(x_{i}\right)$ is incremented by $k$. After a fixed number of conflicts, the activity of literals is divided by a small constant as it was first done in Chaff [14].

$/ / \operatorname{Vars}(M(\boldsymbol{p}), n)$ denotes the set of variables of last $n$ clauses of $M(\boldsymbol{p})$. assignment_to_fix1 $(F, M(\boldsymbol{p}))$

$\{$ act_lit $=$ most_active $(\operatorname{Vars}(M(\boldsymbol{p}), n)$;

return(satisfying_assgn(act_lit)); \}

assignment_to_fix2 $(F, M(\boldsymbol{p}))$

$\{C=$ find_derived_clause $(F, M(\boldsymbol{p}))$;

if $(C==n i l)$ return $($ assignment_to-fix $1(F, M(\boldsymbol{p}))) ; / /$ no conflict clauses in $M(\boldsymbol{p})$

act_lit $=$ most_active $(\operatorname{Vars}(C))$;

return $($ satisfying_assgn $($ act_lit $)) ;\}$

Figure 2. Pseudo-code of the two decision-making procedures of $F I$

In the experiments described in this paper we used two decision making procedures. Their pseudo-code is shown in Figure 2. The first procedure (assignment_to_fix1) just finds the assignment satisfying the most active literal among the variables of clauses from $M(\boldsymbol{p})$ (obviously, only variables whose values are not fixed yet are considered). The number of clauses in $M(\boldsymbol{p})$ may be large which may slow down decision making. To solve this problem we consider only the last $n$ clauses of $M(\boldsymbol{p})$ i.e. the last $n$ entries of $M(\boldsymbol{p})$. In the experiments $n$ was set to 32 .

The second procedure (assignment_to_fix2) is BerkMin-like. First it looks for the conflict clause $C$ of $M(\boldsymbol{p})$ that was derived the most recently. If all conflict clauses are satisfied by $\boldsymbol{p}$, it calls the first decision making procedure i.e assignment_to_fix1. Otherwise, it finds the 
assignment satisfying the most active literal among variables of $C$ whose values have not been fixed yet.

\subsection{BCP procedure and conflict clause generation}

The BCP procedure of $F I$ is almost identical to that of a generic DPLL based SAT-solver. The only difference is that after making a fixed assignment that disagrees with $\boldsymbol{p}$, the latter changes and the set $M(\boldsymbol{p})$ is recomputed. The pseudo-code of a generic BCP procedure (it does not use the speed-up scheme introduced in [20] and further improved in [14]) is shown in Figure 3.

$B C P(F, \boldsymbol{p}, M(\boldsymbol{p})$, assgn $)$

\{ initialize(assgn_queue,assgn);// initialize assignment queue with assgn

// assignment loop

while (assgn_queue is not empty)

$\{$ next_assgn $=$ extract_assignment $($ assgn_queue $) ; / /$ extract next assignment

// let next_var denote the variable to be assigned by next_assgn

for every clause $C$ having variable next_var

\{if (next_assgn satisfies $C$ ) mark_as_satisfied $(C)$;

else if (unit $(C)) / /$ if next_assgn falsifies a literal of $C$ and it became unit

add_new_assgn (C,assgn_queue) $\} / /$ add the assignment satisfying $C$ to the queue

if (next_assgn agrees with $\boldsymbol{p}$ ) continue;

else // next_assgn does not agree with $\boldsymbol{p}$

$\{$ flip_value (next_var, $\boldsymbol{p}) ; / /$ flip the assignment of next_var in $\boldsymbol{p}$

recompute $($ next_var, $M(\boldsymbol{p})) ;\}\}\} / /$ recompute the set of falsified clauses

Figure 3. Pseudo-code of the BCP procedure of $F I$

In contrast to the decision making of FI, its BCP procedure is global. As described above, in its decision making, FI fixes only assignments of variables that occur in clauses of $M(\boldsymbol{p})$. In the BCP procedure, $F I$ keeps track of all the unit clauses regardless of whether they are falsified or satisfied by $\boldsymbol{p}$ and so regardless of their presence in $M(\boldsymbol{p})$. (In terms of fixed assignments, a clause is unit, if all its literals but one are set to 0 by fixed assignments.)

Let $\boldsymbol{p}^{\prime}$ be the point obtained from $\boldsymbol{p}$ by flipping the value of next_var (when next_assgn does not agree with $\boldsymbol{p})$. The set $M\left(\boldsymbol{p}^{\prime}\right)$ is obtained by re-computation of the set $M(\boldsymbol{p})$ in two steps. First, all the clauses of $M(\boldsymbol{p})$ having variable next_var are removed from $M(\boldsymbol{p})$. Second, all the clauses of $F$ that have the literal of next_var falsified by the new assignment to this variable are examined. Every clause of this set that is falsified by the new complete assignment is added to $M(\boldsymbol{p})$.

FI employs a traditional conflict analysis and first-UIP conflict clause generation whose description can be found in [19, 21]. 


\subsection{Initial point generation and restarts}

Currently FI uses the following procedure for generation of initial point $\boldsymbol{p}$. This procedure makes an assignment to a variable $x_{i}$ of the formula and runs BCP procedure to derive all the implied assignments. If implied assignments to a variable contradict each other, one of them is picked randomly. (The contradiction of implied assignments means that the set of assignments made so far can not be extended to a satisfying assignment. In case of a conflict, the DPLL procedure backtracks, but FI's goal here is to build a complete assignment. For this reason, FI continues making assignments even if a conflict occurs.) This goes on until all variables are assigned. This procedure may vary in how variable $x_{i}$ and its assignment are chosen. Variable $x_{i}$ can be chosen randomly or according to a particular order. An assignment to $x_{i}$ can be also picked randomly or according to some heuristic.

We use BCP when generating an initial point $\boldsymbol{p}$ to minimize the size of $M(\boldsymbol{p})$. The reason is twofold. First, by reducing the size of $M(\boldsymbol{p})$ we make all operations involving $M(\boldsymbol{p})$ more efficient. Second, by minimizing the size of $M(\boldsymbol{p})$ we make the decision making of FI more precise. This part is explained in more detail in Subsection 4.1.

FI uses occasional restarts as suggested in [10]. (I.e. once in a while, FI abandons the current search tree to start a new one.) After a restart, FI inherits the last complete assignment $\boldsymbol{p}$ obtained before abandoning the previous search tree. In experiments, $F I$ performed a restart every 150 conflicts.

\section{Proof encryption semantics}

As mentioned in the introduction, one can give two interpretations of $F I$. In the previous section we described FI in terms of the first interpretation. It views $F I$ as a regular SAT-solver with clause learning that maintains a complete assignment to direct its decision-making. The advantage of this interpretation is that FI's operation is very easy to understand. The problem is that this interpretation is based on enumerative semantics. From the viewpoint of this semantics it is very hard to understand why FI should work at all. Indeed, the set of variables that can be assigned a fixed value is specified by a complete assignment i.e. by a minuscule piece of the search space. In this section, we describe and justify an alternative interpretation of $F I$. In this interpretation, $F I$ does not enumerate the search space, but rather visits a set of points that encrypts a resolution proof. The latter is built simultaneously with its encryption.

This section is structured as follows. In subsection 3.1, we recall some basic definitions of the resolution proof system. Subsection 3.2 introduces procedure $S A T(T, F)$ that checks if a set of points $T$ encrypts a resolution proof that $F$ is unsatisfiable. In Subsection 3.3, we show that after a natural extension, the set of points visited by $F I$ contains a point image of the proof built by FI. This result substantiates interpretation of $F I$ in terms of proof encryption semantics. Subsection 3.4 shows that the $S A T(T, F)$ procedure (and more generally, proof encryption semantics) can be used to measure the quality of a set of points visited by a SAT-solver. 


\subsection{Resolution proofs}

Here we recall basic definitions of the resolution proof system [1]. Let $C_{1}$ and $C_{2}$ be two clauses that have opposite literals of a variable $x_{i}$. Then the clause consisting of all the literals of $C_{1}, C_{2}$ except those of $x_{i}$ is called the resolvent of $C_{1}, C_{2}$. (For example if $C_{1}=x_{1} \vee x_{3} \vee x_{5}, C_{2}=x_{2} \vee \overline{x_{3}} \vee x_{7}$, the resolvent of $C_{1}$ and $C_{2}$ is the clause $x_{1} \vee x_{5} \vee x_{2} \vee x_{7}$.) The resolvent of $C_{1}, C_{2}$ is said to be obtained by the resolution operation in variable $x_{i}$.

The resolvent of $C_{1}, C_{2}$ is implied by $C_{1} \wedge C_{2}$. So, if an empty clause is derived from clauses of $F$, then $F$ implies an empty clause and so $F$ is unsatisfiable. Hence, the resolution system is sound. It is also complete, that is, given an unsatisfiable CNF formula $F$, one can always generate a sequence of resolution operations resulting in producing an empty clause. This sequence of operations is called a resolution proof. The resolution proof system is very important from a practical point of view because the best SAT-solvers for solving "industrial" formulas are based on resolution.

\subsection{Procedure $S A T(T, F)$}

In this subsection, we introduce a procedure $S A T(T, F)$. This procedure is not practical (for the reason explained below) and we introduce it just to formalize the notion of a set of points $T$ encrypting a proof. Namely, $T$ encrypts a resolution proof that $F$ is unsatisfiable, if $S A T(T, F)$ terminates with the answer 'unsatisfiable'.

Proof encryption is discussed more thoroughly in [7] using the notion of a stable set of points [6]. In particular, in [7], we explain why one needs two points to encrypt a resolution operation. (If $C$ is the resolvent of clauses $C^{\prime}$ and $C^{\prime \prime}$, then to prove $C^{\prime} \wedge C^{\prime \prime} \rightarrow C$ it is sufficient to build a stable set of two points.) Besides, in [7], we describe how one can encrypt a resolution proof in such a way that the latter can be efficiently recovered. The procedure $S A T(T, F)$ described below is an adaptation of procedure $S A T(T, F, L)$ of [7] for the purpose of this paper. The full discussion of this topic is beyond the scope of this paper.

The pseudo-code of $S A T(T, F)$ is given in Figure 4. First, $S A T(T, F)$ checks if a point $\boldsymbol{p}$ of $T$ satisfies $F$. If such a point exists, then $S A T(T, F)$ returns 'satisfiable'. Then in the while loop, $S A T(T, F)$ generates resolvents specified by $T$ in topological order i.e. level by level. Clauses of the original formula $F$ have level 0 . If $C$ is the resolvent of clauses $C^{\prime}$ and $C^{\prime \prime}$, then level $(C)=\max \left(\right.$ level $\left(C^{\prime}\right), \operatorname{level}\left(C^{\prime \prime}\right)+1$. The $S A T(T, F)$ procedure generates the resolvent $C$ of clauses $C^{\prime}, C^{\prime \prime}$ of the current formula $F$, only if $T$ contains points $\boldsymbol{p}^{\prime}$ and $\boldsymbol{p}^{\prime \prime}$ such that the following two conditions hold:

- $C^{\prime}\left(\boldsymbol{p}^{\prime}\right)=0$ and $C^{\prime \prime}\left(\boldsymbol{p}^{\prime \prime}\right)=0$.

- $C\left(\boldsymbol{p}^{\prime}\right)=0$ and $C\left(\boldsymbol{p}^{\prime \prime}\right)=0$.

The points $\boldsymbol{p}^{\prime}$ and $\boldsymbol{p}^{\prime \prime}$ are called a point image of the resolution operation over clauses $C^{\prime}$ and $C^{\prime \prime}$. In other words, $S A T(T, F)$ generates a resolvent $C$ only if $T$ contains a point image of the corresponding resolution operation. We also assume that the resolvent $C$ is generated only if it is not implied by some existing clause of $F$. In other words, we assume that $S A T(T, F)$ generates only new clauses. Let $F_{1}$ denote the set of resolvents generated at the current topological level. If $F_{1}=\emptyset$ (i.e. no new resolvents were generated), $\operatorname{SAT}(T, F)$ returns 'unknown'. This outcome means that $T$ contains an insufficient number of points. 
If $F_{1}$ contains an empty clause, $S A T(T, F)$ returns 'unsatisfiable'. Otherwise, the clauses of $F_{1}$ are added to $F$ and $S A T(T, F)$ starts building a new topological level of the proof.

$/ / T$ is a set of points, $F$ is a CNF formula

$\operatorname{SAT}(T, F)$

$\{$ if $(F(\boldsymbol{p})=1$ for a point $\boldsymbol{p}$ of $T)$ return('satisfiable');

while (true)

$\left\{F_{1}=\right.$ generate_next_level_resolvents $(T, F)$;

if $\left(F_{1}==\emptyset\right)$ return('unknown');

if ( $F_{1}$ contains an empty clause) return('unsatisfiable');

$\left.\left.F=F \cup F_{1} ;\right\}\right\}$

Figure 4. Pseudo-code of $S A T(T, F)$

Let $R$ be a resolution proof that a CNF formula $F$ is unsatisfiable. Let $T$ be a set of points that has the following property. For any resolvent $C$ of $R$ obtained from parent clauses $C^{\prime}$ and $C^{\prime \prime}$ there are two points $\boldsymbol{p}^{\prime}, \boldsymbol{p}^{\prime \prime}$ of $T$ forming a point image of the resolution operation over $C^{\prime}$ and $C^{\prime \prime}$. Then the set $T$ is called a point image of resolution proof $\boldsymbol{R}$. From definitions of $S A T(T, F)$ and point image it follows that $S A T(T, F)$ returns 'unsatisfiable' if and only if the set $T$ contains a point image of a resolution proof that $F$ is unsatisfiable. One can view a point image $T$ of a proof $R$ as an encryption of $R$, while $S A T(T, F)$ can be viewed as a procedure for recovering $R$ from $T$.

It is not hard to show that a proof $R$ has a point image whose size is at most twice the size of the proof $R$ (in terms of resolution operations). Indeed, if $C$ is a resolvent of $R$ obtained from clauses $C^{\prime}$ and $C^{\prime \prime}$, one can always find points $\boldsymbol{p}^{\prime}, \boldsymbol{p}^{\prime \prime}$ forming a point image of this resolution operation. The union of point images of all resolution operations of $R$ is a point image of $R$. Obviously, the size of such a point image is at most twice the size of $R$.

Note that one can not guarantee that $S A T(T, F)$ will recover $R$ from a point image $T$ in polynomial time (which makes $S A T(T, F)$ impractical). Even though $S A T(T, F)$ imposes restrictions on generated resolvents, their number still can be prohibitively large. However, since we do not use $S A T(T, F)$ for real computation it does not matter much. Our goal here is twofold. First, we want to show how one can encrypt a resolution proof using complete assignments. Second, we use $S A T(T, F)$ to measure the quality of a set of points visited by a SAT-solver (see Subsection 3.4). So, in a sense, we only use the fact that for some sets of points, $S A T(T, F)$ results in generating an empty clause, while for others it stops before generating such a clause.

\subsection{FI builds a point image of the proof it generates}

Let $F$ be an unsatisfiable formula and $R$ be a resolution proof found by $F I$ when solving $F$. Let $T$ be the set of points visited by FI. In this subsection, we show that after a natural extension of the set $T$, it becomes a point image of $R$. In Subsection 3.3.1, we describe this extension and in subsection 3.3.2 we prove that the extended set $T$ is a point image of $R$. Importantly, the fact that the set of visited points forms an image of the proof $F I$ 
builds does not mean that FI does anything "special". This fact just implies that the proof encryption semantics agrees with a resolution based SAT-solver using clause learning.

\subsubsection{EXTENSION OF THE SET OF VISITED POINTS}

Denote by $T$ the set of points visited by $F I$ when generating a proof $R$. We need to extend $T$ because during BCP FI may fix assignments of variables of clauses that are not in $M(\boldsymbol{p})$. This extension of $T$ is natural in the sense that the only reason why $F I$ does not visit "explicitly" a point added by the extension is that this point can not be a satisfying assignment. Let $C$ be a unit clause (i.e all the literals of $C$ but one are set to 0 by fixed assignments). Suppose, the assignment satisfying $C$ agrees with the current complete assignment $\boldsymbol{p}$. (So $C$ is not $M(\boldsymbol{p})$.) After this assignment is fixed by $F I$, the clause $C$ may get involved in a conflict without being falsified by any point of $T$. However, the necessary condition for $T$ to be a point image of $R$ is that every clause of $R$ is falsified by a point of $T$. So, obviously, $T$ is not an image of $R$.

Suppose we extend $T$ by the set of points that falsify all clauses that became unit during a run of $\mathrm{BCP}$ and from which an (implied) assignment was derived. This extension can be built in the following way. If, during BCP a unit clause $C$ is satisfied by fixing an assignment to a variable $x_{i}$ and this assignment agrees with the current point $\boldsymbol{p}$, we add to $T$ the point $\boldsymbol{p}^{\prime}$ obtained from $\boldsymbol{p}$ by flipping the value of $x_{i}$. Obviously, $\boldsymbol{p}^{\prime}$ falsifies $C$. Note that each added point is at Hamming distance one with an existing point of the current set $T$. The size of the extended set is no more than $n$ times the size of the original set $T$.

\subsubsection{Extended Set of points is an Image of the PRoOf BUilt By FI}

Let $F I$ encounter a conflict and $C_{c n f l}$ be the conflict clause produced in this conflict. The conflict clause generation based on the first-UIP scheme (that is used by $F I$ ) is well described in [21]. According to this scheme, $C_{c n f l}$ is generated by resolving clauses from which assignments were deduced at the conflict decision level and the clause that became unsatisfiable. Let $C_{c n f l}$ be obtained by resolving clauses $C_{1}, . ., C_{k}$ of the current CNF formula $F$. Here $C_{1}, . ., C_{k-1}$ are clauses from which assignments were deduced during $\mathrm{BCP}$ at the conflict level and $C_{k}$ is the unsatisfiable clause. (Note that during BCP at the conflict level, assignments may have been deduced from clauses other than $C_{1}, \ldots, C_{k-1}$. But we are interested only in the clauses involved in the conflict.) Assume that $C_{1}, . ., C_{k-1}$ are numbered in the order in which they were processed by BCP (that is if $i<j$, then an assignment was deduced from $C_{i}$ before deduction from $C_{j}$ ).

In the first-UIP scheme, $C_{c n f l}$ is obtained from $C_{1}, . ., C_{k}$ by resolving them in the "reverse order". That is, first, $C_{k}$ is resolved with $C_{k-1}$. Then $C_{k-2}$ is resolved with the resolvent of $C_{k}$ and $C_{k-1}$ and so on.

Proposition. The extended set of points $T$ is a point image of the proof generated by FI.

Proof. We need to show that for each of $k$-1 resolution operations performed to generate $C_{c n f l}$, the extended set $T$ contains two points forming a point image of this resolution operation. This can be proved by induction in the number of resolutions. Note that by definition of the conflict situation, the clause $C_{k}$ (i.e. the clause that became unsatisfiable) had to be falsified by the current complete assignment (denote it by $\boldsymbol{p}_{\text {cnfl }}$ ). 


\section{E. GoldBerG}

Basis. The fact that $T$ contains a complete assignment $\boldsymbol{p}_{c n f l}$ falsifying $C_{k}$ and that the variables of $\operatorname{Vars}\left(C_{k}\right)$ have fixed assignments is the basis of our inductive proof.

Denote by $R_{m}$ the clause equal to the unsatisfiable clause $C_{k}$ for $m=1$ or to the resolvent of clause $C_{k-(m-1)}$ and clause $R_{m-1}$ (obtained by resolving clauses $C_{k}, C_{k-1}, . ., C_{k-(m-2)}$ ) for $m>1$.

The inductive statement of the proof is as follows. We assume that $R_{m}$ is falsified by $\boldsymbol{p}_{\text {cnfl }}$ and all the variables of $\operatorname{Vars}\left(R_{m}\right)$ have assignments fixed before an assignment was deduced from clause $C_{k-(m-1)}$.

Using this assumption we will show that

1. The inductive statement holds for the next value of $m$.

2. $T$ contains a point $\boldsymbol{p}_{m}$ falsifying the clause $C_{k-m}$ such that $\boldsymbol{p}_{c n f l}$ and $\boldsymbol{p}_{m}$ form a point image of the resolution operation over $R_{m}$ and $C_{k-m}$ producing clause $R_{m+1}$.

Proof of the first condition. Denote by Ded_var $\left(C_{k-m}\right)$ the variable whose value was deduced from $C_{k-m}$ during BCP. Note that before a value of $\operatorname{Ded} \_v a r\left(C_{k-m}\right)$ was deduced from $C_{k-m}$, the literals of the other variables of $C_{k-m}$ were set to 0 by fixed assignments. So these literals are set to 0 by $\boldsymbol{p}_{\text {cnfl }}$ too. Then, taking into account that $\boldsymbol{p}_{\text {cnfl }}$ falsifies $R_{m}$ we conclude that the clause $R_{m+1}$ is falsified by $\boldsymbol{p}_{\text {cnfl }}$. Note that the assignments to the variables of $\operatorname{Vars}\left(C_{k-m}\right) \backslash\left\{\operatorname{Ded}_{-} \operatorname{var}\left(C_{k-m}\right)\right\}$ were fixed before deducing a value of Ded_var $\left(C_{k-m}\right)$. So all the literals of $R_{m+1}$ were fixed at 0 before derivation of $\operatorname{Ded} \_v a r\left(C_{k-m}\right)$.

Proof of the second condition. Let $\boldsymbol{p}$ be the point that was the current complete assignment of $F I$ at the time an assignment was deduced from clause $C_{k-m}$. Denote by $\boldsymbol{p}_{m}$ the point of $T$ defined as follows. If $C_{k-m}$ was falsified by $\boldsymbol{p}$, then $\boldsymbol{p}_{m}=\boldsymbol{p}$. If $C_{k-m}$ was satisfied by $\boldsymbol{p}$, then $\boldsymbol{p}_{m}=\boldsymbol{p}^{\prime}$ where $\boldsymbol{p}^{\prime}$ is obtained from $\boldsymbol{p}$ by the extension of $T$ described above (i.e. by flipping the value of variable $\operatorname{Ded} \_v a r\left(C_{k-m}\right)$ ). In either case, $C_{k-m}\left(\boldsymbol{p}_{m}\right)=0$.

Now we need to show that both $\boldsymbol{p}_{m}$ and $\boldsymbol{p}_{c n f l}$ falsify the resolvent $R_{m+1}$ of $C_{k-m}$ and $R_{m}$ (and so $\boldsymbol{p}_{m}$ and $\boldsymbol{p}_{c n f l}$ form a point image of the resolution operation over $C_{k-m}$ and $R_{m}$.) We already showed above that $\boldsymbol{p}_{c n f l}$ falsifies $R_{m+1}$. Now we show that $\boldsymbol{p}_{m}$ sets to 0 all the literals of $R_{m}$ (except maybe the literal of Ded_var $\left(C_{k-m}\right)$ ) and hence, taking into account that $C_{k-m}\left(\boldsymbol{p}_{m}\right)=0$, the point $\boldsymbol{p}_{m}$ falsifies $R_{m+1}$. Let lit $\left(x_{i}\right)$ be a literal of $R_{m}$ (where $x_{i}$ is different from Ded_var $\left(C_{k-m}\right)$ ). Assume the contrary i.e. that the value of $x_{i}$ in $\boldsymbol{p}_{m}$ sets lit $\left(x_{i}\right)$ to 1 and so $\boldsymbol{p}_{m}$ satisfies the resolvent $R_{m}$. This assignment to $x_{i}$ can not be fixed because then $\boldsymbol{p}_{c n f l}$ would have the same value of $x_{i}$ and so it would satisfy $\boldsymbol{R}_{m}$. So the opposite value of $x_{i}$ must have been deduced later. This value of $x_{i}$ could not have been deduced from $C_{k-m}$ (because $x_{i}$ is different from $\operatorname{Ded} d_{-} v a r\left(C_{k-m}\right)$ ) or $C_{k-(m-1)}$ (or from any clause $\left.C_{k-(m-p)}, p>1\right)$ because all assignments to the variables of $R_{m}$ were fixed before derivation from $\left.C_{k-(m-1)}\right)$. This implies that there was one more derivation between $C_{k-m}$ and $C_{k-(m-1)}$. But this contradicts our assumption that the sequence $C_{1}, \ldots, C_{k}$ lists all the clauses involved in the conflict in the order in which assignments were deduced from them by BCP. 


\subsection{Using proof encryption semantics to measure quality of sets of points}

One of the main problems of the enumerative semantics is as follows. Let $T^{i}$ and $T^{j}$ be two sets of points which have been proved not to contain a satisfying assignment. Enumerative semantics essentially implies that if $\left|T^{i}\right|>\left|T^{j}\right|$ then $T^{i}$ is more "valuable" than $T^{j}$. This makes perfect sense if the search space is partitioned into non-overlapping subsets $T^{i}$. However this is not true, for example, for SAT-solvers with clause learning where subspaces $T^{i}$ corresponding to leaf nodes may overlap. In such a case, the most important quality of subspaces $T^{i}$ is not their size but their synergy i.e. how well they fit each other to produce an empty clause as a proof of unsatisfiability.

The proof encryption semantics gives a way to measure the quality of a set of visited points. We illustrate this claim by two examples below.

Suppose that a CNF formula $F$ is equal to $F^{\prime} \wedge\left\{x_{i}\right\}$ where $x_{i}$ is just a unit clause. From the viewpoint of enumerative semantics setting $x_{i}$ to 1 (that would be done by a DPLL-like SAT-solver to satisfy the unit clause $x_{i}$ ) rules out half the search space. So one has to conclude that $50 \%$ of work has been done. On the other hand, obviously, the complexity of proving that $F\left(x_{i}=1\right)$ is unsatisfiable may be arbitrary high.

From the viewpoint of the proof encryption semantics, the situation is different. Let $R$ be a "reasonable" resolution proof that $F$ is unsatisfiable. By a reasonable proof we mean one that first derives clause $\overline{x_{i}}$ from $F^{\prime}$ and then resolve $x_{i}$ and $\overline{x_{i}}$ to produce an empty clause. (This is exactly the kind of a proof a state-of-the-art SAT-solver with clause learning would build). If one constructs a point image of such a proof, only one point of this image has to falsify $x_{i}$ (namely a point of the image of the resolution operation over clauses $x_{i}$ and $\overline{x_{i}}$.) So in the proof encryption semantics, the work done by ruling out the subspace $x_{i}=0$ amounts to gaining only one point of the image of a future proof.

Let us consider another example. Suppose an unsatisfiable CNF formula $F$ contains a clause $C=x_{i} \vee x_{m}$. Suppose that $C$ is irredundant i.e. removing $C$ from $F$ makes the latter satisfiable. Then $C$ has to be a part of any resolution proof $R$ that $F$ is unsatisfiable. Let us $T^{\prime}$ be the set of complete assignments falsifying $C$ (i.e. $T^{\prime}$ consists of all complete assignments with $x_{i}=0$ and $x_{m}=0$ ). Although $T^{\prime}$ makes up a quarter of the search space, it does not contain a point image of a resolution proof (because $T^{\prime}$ can not contain point images of operations resolving clauses in $x_{i}$ or $x_{m}$ ). On the other hand, there might be a dramatically smaller set of points $T^{\prime \prime}$ that contains a point image of a proof.

\section{Complete assignments contain more information about the formula than partial assignments}

In this section, we compare partial and complete assignments at a different angle. A partial assignment specifies more points of the search space, but it contains much less information about formula $F$ (because a partial assignment "sees" only a fraction of variables of $F$ ). On the contrary, a complete assignment specifies only one point $\boldsymbol{p}$ of the search space but contains more information about $F$. In Subsection 4.1 we show that this advantage of a complete assignment can be used for identifying small unsatisfiable cores. Subsection 4.2 demonstrates how greater informational capacity of complete assignments can be used for solving satisfiable formulas. 


\subsection{Identification of unsatisfiable cores}

Let $F$ be a CNF formula that can be represented as $F_{1} \wedge F_{2}$ where $F_{1}$ is a large CNF formula (either satisfiable or unsatisfiable) that is very hard to solve and $F_{2}$ is a small unsatisfiable CNF formula. Assume for the sake of simplicity, that the variables of $F_{1}$ and $F_{2}$ do not overlap. Since $F_{1}$ is much larger than $F_{2}$, first conflict clauses derived by a SAT-solver will depend on variables of $F_{1}$. Indeed, suppose, for example, that $\left|\operatorname{Vars}\left(F_{2}\right)\right|=10$ and $\left|\operatorname{Vars}\left(F_{1}\right)\right|=10^{6}$. Suppose that to get a conflict in $F_{2}$ one has to assign at least 5 variables of $F_{2}$. Then the probability of producing a conflict in $F_{2}$ is roughly speaking $\left(10^{-6}\right)^{5}$ i.e. $10^{-30}$. (Of course we assume here that the clauses derived from conflicts in $F_{1}$ are not too long. Otherwise we would need to take into account that a SAT-solver has to make many assignments in $F_{1}$ before a conflict occurs. This increases the chances of producing a conflict in $F_{2}$.) So with great probability conflicts will be generated in the formula $F_{1}$. Then the activity of variables of $F_{1}$ will be growing (we assume that our SAT-solver uses $Z$ chaff-like decision making). This will force the SAT-solver to keep branching on variables of $F_{1}$ until an assignment satisfying $F_{1}$ is produced or $F_{1}$ is proved unsatisfiable.

Let us consider solving the formula above by FI. No matter what the current complete assignment $\boldsymbol{p}$ is, it falsifies a clause of $F_{2}$ (because the latter is unsatisfiable). Then, although the choice of branching variables reduces to $\operatorname{Vars}(M(\boldsymbol{p}))$ (i.e. to the variables of clauses falsified by $\boldsymbol{p}$ ), a variable of $F_{2}$ is always available for branching and so good choices remain intact. Moreover, since the set $\operatorname{Vars}(M(\boldsymbol{p}))$ is much smaller than $\operatorname{Vars}(F)$, finding a variable of $F_{2}$ becomes dramatically easier.

One may think that the case when a CNF formula has a small unsatisfiable core is relatively rare. However, even if the original CNF formula $F$ is irredundant (i.e. no clause of $F$ can be removed without making $F$ satisfiable), it becomes redundant after adding conflict clauses and/or making decision assignments. This may lead to appearance of small unsatisfiable cores. So the problem of finding such cores is ubiquitous in resolution based SAT solving.

Interestingly, the reasoning above implies that to make identification of an unsatisfiable core easier one should keep the size of the set $M(\boldsymbol{p})$ small. One can view variables $\operatorname{Vars}\left(F_{2}\right)$ of the small unsatisfiable core $F_{2}$ as "signal" and variables $\operatorname{Vars}\left(F_{1}\right)$ of $F_{1}$ as "noise". No matter how FI picks $\boldsymbol{p}$, a signal variable will be in $\operatorname{Vars}(M(\boldsymbol{p}))$. On the other hand, by minimizing the size of $M(\boldsymbol{p}), F I$ may considerably reduce the number of noise variables and so improve the signal/noise ratio in $\operatorname{Vars}(M(\boldsymbol{p}))$. In particular, when generating an initial point $\boldsymbol{p}$ (Subsection 2.6) FI tries to minimize the size of $M(\boldsymbol{p})$.

\subsection{Storing information about previous assignments when solving satisfiable formulas}

Greater informational "capacity" of complete assignments can be also leveraged when solving satisfiable formulas. A DPLL-like SAT-solver operating on complete assignments loses information about assignments made before backtracking (although some part of this information is represented implicitly in the learned clauses). Besides, to find a satisfying assignment such a SAT-solver has to keep extending the current partial assignment until all clauses are satisfied. On the contrary, when backtracking, FI preserves more information due to maintaining a complete assignment (that remembers the last assignments made be- 
fore backtracking). Besides, FI does not have to satisfy all the clauses by fixed assignments. It stops as soon as the current complete assignment $\boldsymbol{p}$ satisfies all the clauses (at this time, only a small fraction of assignments of $\boldsymbol{p}$ may be fixed).

\section{Background}

In this section, we compare $F I$ with other SAT-solvers and give some background information. As mentioned above, $F I$ has two different interpretations. In the first interpretation, FI should be compared with other DPLL-like SAT-solvers like Zchaff [14], BerkMin [8], Siege, Minisat [4]. (In this interpretation, FI is a DPLL-like SAT-solver with clause learning whose choice of branching variables is limited to $\operatorname{Vars}(M(\boldsymbol{p})))$. The main difference of $F I$ from these SAT-solvers is in decision making. The decision making driven by conflict activity of literals (introduced by $Z$ chaff) is very slow for large formulas if one has to examine all free (i.e. unassigned) variables. A solution of BerkMin was to branch on variables of the most recently derived conflict clause that was still unsatisfied. This considerably reduced the number of variables to examine. (A similar solution was independently introduced in Siege.) The flaw of this approach is that if the current CNF formula does not have any unsatisfied conflict clauses, BerkMin still has to pick an assignment out of the entire set of free variables. (As we show in Section 6 this drawback slows down the performance of BerkMin for large BMC formulas.)

Minisat took a slightly different approach. The activity of variables is computed in Minisat similarly to BerkMin. Like BerkMin it is more aggressive than Zchaff in giving preference to recently derived clauses. However, in contrast to BerkMin and Siege, Minisat still looks for the most active variable among all the free ones. It does this efficiently by maintaining a heap of variable activities. FI suggests a new way to speed up decision making. It reduces the choice of available branching variables to those that are falsified by the current complete assignment.

In the second interpretation, $F I$ is a resolution based algorithm with clause learning that operates on complete assignments. From this point of view FI should be compared with algorithms operating on complete assignments pioneered in $[16,17]$. One can classify these algorithms into three groups: incomplete stochastic local search algorithms, hybrid algorithms combining DPLL and local search and local search algorithms that can solve unsatisfiable formulas. The first group comprises of algorithms like gsat [16], walksat [17], unitwalk [12]. Although FI bears some similarity to walksat (they both maintain the set $M(\boldsymbol{p})$ of clauses falsified by the current complete assignment), the main difference of $F I$ from the algorithms of this group is that it is complete. So FI can escape local minima that may trap a local search algorithm. On the other hand, being a DPLL-like procedure, FI does not work well for classes of formulas like satisfiable random formulas where local search algorithms show strong performance.

The second group consists of algorithms like [13, 11]. In [13], in every node of the DPLL procedure, a local search procedure is invoked to identify the next variable to branch on. In [13], an important observation was made that local search can be used for identifying an unsatisfiable core of the initial formula. The approach of [13] was also tried in [11] with the following modification. Before running a local search procedure at a node of the search tree, dependencies between variables of the current formula were computed. The 
difference of $F I$ from the algorithms of this group is twofold. First, FI uses clause learning and decision making based on computing conflict activity of literals and so it can be applied to solving very large CNF formulas. Second, algorithms of this group consider local search procedures and DPLL procedure as separate entities. On the contrary, in FI these two kinds of procedures are merged together into a single DPLL-like procedure operating on complete assignments.

There are very few local search procedures that can be applied to solving unsatisfiable formulas. A complete local search algorithm augmented by clause generation was introduced in [5]. Clauses were generated in the algorithm of [5] in "a mechanical way" just to escape local minima. Our experiments showed that the SAT-solver of [5] generates an enormous number of new clauses and so fails to prove the unsatisfiability of even very small CNF formulas. In [18], the idea of proving unsatisfiability by local search was introduced. The suggestion was to encrypt proofs in some way and then look for proof encryptions by a stochastic local search procedure. This idea was implemented in [15] but the suggested algorithm performed well only on a fraction of CNF formulas. Since FI is based on proof encryption semantics one can view it as an implementation of the elegant idea of [18]. However, in contrast to [18], FI is a complete algorithm that builds a proof encryption along with the proof itself. Such a strategy makes sense because, intuitively, an encryption that is good for one formula may not work well for another.

\section{Experimental Results}

In this section, we give results of some experiments with an implementation of FI. Experiments were run on Intel's Xeon CPU $(3.06 \mathrm{GHz})$ under Linux. The main objective of the experiments was to show that although $F I$ was very limited in the choice of branching variables, it was still competitive with state-of-the-art SAT-solvers in the number of backtracks (conflicts). So we used a very simple implementation that lacked the techniques commonly employed to speed up a SAT-solver. Besides, we tried to keep our implementation of FI as simple as possible (to facilitate changing the code of $F I$ ). Nevertheless we give some results of $F I$ performance showing that even our primitive implementation is competitive with highly optimized SAT-solvers for large Bounded Model Checking (BMC) formulas. In our experiments, we used the second decision-making heuristic of Subsection 2.4 for the formulas of Tables 1, 7 (it was slightly modified for the formulas of Table 7 as described below). For the rest of the formulas we used the first decision-making heuristic of subsection 2.4 with $n=32$ (i.e. $F I$ took into account only last 32 entries of the set $M(\boldsymbol{p})$ ). In all the experiments, a restart was performed every 150 conflicts.

This section is structured as follows. In Subsection 6.1 we briefly describe the implementation we used in experiments. Subsection 6.2 compares FI with Zchaff, Minisat and BerkMin. In Subsection 6.3, we show that FI can not be efficiently simulated by a SATsolver that reduces choices by picking the next branching variable from a random window of variables. Finally, Subsection 6.4 gives some results on testing the satisfiability of formulas with small unsatisfiable cores. 


\subsection{A brief description of the implementation we used in experiments}

FI was implemented in $\mathrm{C}++$ using the gcc compiler (version 3.2.2). We used the STL library for data structures like dynamic arrays. As mentioned above, our implementation of $F I$ was very simple. It did not have advanced features like fast BCP, efficient formula representation, special treatment of binary clauses, removal of inactive conflict clauses and so on. In our implementation of the BCP procedure, to check if a clause was unit FI just counted the number of unassigned literals (as it was done, for example, in Grasp [19]). Instead of "watching" literals, for every literal lit $\left(x_{i}\right), F I$ maintained the list of clauses of the current formulas having lit $\left(x_{i}\right)$. So when lit $\left(x_{i}\right)$ was set to $0, F I$ just needed to examine the clauses of the corresponding list to see if new unit clauses appeared. To avoid examining satisfied clauses, when lit $\left(x_{i}\right)$ was set to 1 , all the clauses with lit $\left(x_{i}\right)$ unsatisfied so far were marked as satisfied. The clauses satisfied at a particular decision level were recorded so that they could be easily unmarked when backtracking.

For more efficient processing of the set $M(\boldsymbol{p})$ of clauses falsified by the current complete assignment $\boldsymbol{p}$, our implementation of $F I$ maintained the following three data structures. An array weight contained the information about variable occurrences in clauses of $M(\boldsymbol{p})$. If weight $[i]=0$, then no clause of $M(\boldsymbol{p})$ contains $x_{i}$. An array Vars contained the set of all variables that were present in $M(\boldsymbol{p})$ (i.e. all variables $x_{i}$ with weight $[i]>0$ ). Finally, FI maintained an array falsified_clauses, where falsified_clauses $[i]$ gave the clauses of $M(\boldsymbol{p})$ having variable $x_{i}$. All three data structures were updated every time, the set $M(\boldsymbol{p})$ changed. The array weights allowed one to efficiently check if $x_{i}$ was in a clause of $M(\boldsymbol{p})$. The array Vars was used to efficiently enumerate all variables of clauses falsifying $\boldsymbol{p}$. The array falsified_clauses was used for updating $M(\boldsymbol{p})$. When the value of variable $x_{i}$ was flipped, the clauses to be removed from $M(\boldsymbol{p})$ were extracted from falsified_clauses $[i]$.

\subsection{Comparison with other SAT-solvers}

In this subsection, we compare our implementation of FI with BerkMin [8], version 561 and Minisat [4], version 1.13. To put FI performance in perspective, we also give the results of Zchaff (version Z2001.2.17 of year 2001). Table 1 compares SAT-solvers for some known benchmark formulas of small and medium size (up to 60 thousand variables). The second column gives the number of formulas in a benchmark set. For every SAT-solver the runtime (in seconds) and number of conflicts is reported. Results of Table 1 show that FI was competitive with BerkMin and Minisat in terms of conflicts (with the exception of the hole formulas where FI had considerably fewer backtracks). FI was better than Zchaff in terms of backtracks (except for the hole formulas where Zchaff had slightly fewer backtracks). As for performance, BerkMin and Minisat were much faster than FI due to their advanced optimization techniques. Zchaff was also faster than FI and only when it had a significantly larger number of backtracks (bmc and $i i$ benchmarks) it was slower than $F I$.

In Table 2, we compare these four SAT-solvers on a set of large BMC formulas (up to a few million variables). These formulas describe various properties of more than a dozen of customer designs. This set consists of 79 formulas (28 satisfiable and 51 unsatisfiable). The formulas were generated by the SMV model-checker [22]. For all the four SAT-solvers, Table 2 gives the number of conflicts (in thousands) and runtime (in hours). (Zchaff and 


\section{E. GoldBerG}

Table 1. Comparison of FI with other SAT-solvers on some known benchmarks

\begin{tabular}{|l|l|l|l|l|l|l|l|l|l|}
\hline \multirow{2}{*}{ Name } & \multirow{2}{*}{ \#inst } & \multicolumn{2}{l|}{ Zchaff-2001 } & \multicolumn{2}{l|}{ BerkMin561 } & \multicolumn{2}{l|}{ Minisat } & \multicolumn{2}{l|}{ FI } \\
\cline { 3 - 11 } & & \#cnfls & $\begin{array}{l}\text { time } \\
(\mathrm{s})\end{array}$ & \#cnfls & $\begin{array}{l}\text { time } \\
(\mathrm{s})\end{array}$ & \#cnfls & time & \#cnfls & time \\
\hline blocksworld & 7 & 15,325 & 9.5 & $\mathbf{4 , 2 3 9}$ & $\mathbf{1 . 6}$ & 4,732 & 1.7 & 7,211 & 25.3 \\
\hline miters & 13 & 124,987 & 7.9 & 31,998 & $\mathbf{1 . 8}$ & 48,118 & 2.7 & $\mathbf{2 8 , 3 8 6}$ & 9.8 \\
\hline bmc & 13 & 309,652 & 523.1 & 53,989 & 54.0 & 44,195 & $\mathbf{2 9 . 8}$ & $\mathbf{4 1 , 8 4 6}$ & 210.4 \\
\hline planning & 6 & 74,639 & 49.2 & 31,238 & 13.4 & $\mathbf{1 7 , 1 5 3}$ & $\mathbf{6 . 0}$ & 22,786 & 159.1 \\
\hline ii & 41 & 75,859 & 53.4 & 6,505 & 0.5 & 4,088 & $\mathbf{0 . 4}$ & $\mathbf{9 0 5}$ & 1.8 \\
\hline hole & 5 & $\mathbf{3 7 , 4 5 2}$ & $\mathbf{1 1 . 3}$ & 224,803 & 32.3 & $1,538,350$ & 33.6 & 48,383 & 159.0 \\
\hline
\end{tabular}

BerkMin were not able to finish 5 and 3 formulas respectively with the timeout limit of 10 hours.)

In terms of performance, $F I$ was considerably faster than Zchaff and BerkMin. FI was faster than Minisat on satisfiable formulas and about two times slower for unsatisfiable formulas. These surprising results can be explained as follows. Using fast BCP is crucial only when a CNF formula has a lot of long clauses. These long clauses may be either present in the initial formula or may appear as conflict clauses. When solving a large BMC formula that initially consists of clauses with no more than three literals, fast BCP becomes beneficial only if the SAT-solver has to generate a lot of conflict clauses. However, if the number of conflicts is relatively small (like 10-20 thousands of conflict clauses for a formula of a few million clauses) the speed-up provided by fast BCP is not so significant. In this situation, efficient and "precise" decision making becomes very important. However, the decision making of all three SAT-solvers that we compared with FI (we briefly described their decision-making in the previous section) has drawbacks.

Zchaff takes a large amount of time to find next branching variable. For smaller formulas this drawback did not show but for large formulas of Table 2 it became a big problem. The decision making of BerkMin is much faster than Zchaff's when the current formula has unsatisfied conflict clauses. However, for large BMC formulas that can be solved without generating too many clauses, BerkMin often had to pick next branching variable out of all free variables. (Version 561 of BerkMin does use a method to reduce time taken by the decision-making procedure, but it was designed for small or medium size formulas.)

Minisat spends much less time on decision making. The problem, however, is in the quality of decision making of Minisat. In general, Minisat had to make many more decisions per conflict than FI, which increased the amount of work done during BCP. (The same applies to Zchaff and BerkMin.)

Tables 3 and 4 give more detailed comparison of Minisat and FI on BMC formulas used in Table 2. Their performance on a sample of these formulas is given in Table 3. The names of satisfiable formulas are marked in the first column of Table 3 with an asterisk. For either SAT-solver, Table 3 gives the number of decision assignments (in thousands), implied assignments (in millions), conflicts (in thousands) and runtime (in seconds). Results for the 
first three formulas (always.100, mcbdm.40, cfet.22) show that FI was faster because it made fewer implied and decision assignments. So even though Minisat is much more optimized than FI, the amount of extra work Minisat had to do slowed it down. For example, for the formula cfet.22, FI made more backtracks $(31,000$ versus 14,000) but considerably fewer decisions (368,000 versus $1,763,000)$ and implied assignments $\left(23 \times 10^{6}\right.$ versus $\left.98 \times 10^{6}\right)$.

For satisfiable formulas, in addition to making more implied and decision assignments, in many cases Minisat had many more backtracks. The reason for the good performance of $F I$ on satisfiable formulas was already mentioned in the introduction and in Section 2. FI reports satisfiability as soon as the set $M(\boldsymbol{p})$ becomes empty. This may happen even if only a fraction of variables have fixed values while the rest of the variables are assigned a value only in $\boldsymbol{p}$. In this case, only a fraction of clauses of the original formula are satisfied by fixed assignments. On the contrary, a traditional SAT-solver operating on partial assignments has to go on making assignments until all the clauses are satisfied by fixed assignments. Needless to say, that such a SAT-solver may still encounter a lot of conflicts before a satisfying assignment is found. For example, for a formula of Table 2 of 700 thousand variables, FI reported satisfiability after 1700 backtracks when only 22,000 variables were assigned fixed values. It took Minisat 420,000 backtracks and more than one hour of runtime to assign the rest of the variables of this formula

In Table 4, we summarize the performance of Minisat and FI on the formulas of Table 2 in terms of implied and decision assignments (given in millions). This table shows that FI made considerably fewer assignments of either kind for both satisfiable and unsatisfiable formulas. Note that this result can be explained by better identification by FI of unsatisfiable cores. After FI has fixed some assignments a small unsatisfiable core may appear in the current formula. The same occurs after Minisat has made some assignments to free variables. However, FI is more likely to pick a core variable than Minisat (see Subsection 4.1). Making assignments to variables outside the core may lead to redundant BCP calls.

Table 2. Comparison of FI with other SAT-solvers on large BMC formulas (timeout 10 hours)

\begin{tabular}{|c|c|c|c|c|c|c|c|c|c|}
\hline \multirow{2}{*}{$\begin{array}{l}\text { Cate- } \\
\text { gory }\end{array}$} & \multirow[t]{2}{*}{ \#inst } & \multicolumn{2}{|c|}{ Zchaff-2001 } & \multicolumn{2}{|c|}{ BerkMin561 } & \multicolumn{2}{|c|}{ Minisat } & \multicolumn{2}{|l|}{$F I$} \\
\hline & & $\begin{array}{l}\# \mathrm{cnfls} \\
\times 10^{3}\end{array}$ & $\begin{array}{l}\text { time } \\
\text { (hrs) } \\
\text { [aborted] }\end{array}$ & $\begin{array}{l}\# \text { cnfls } \\
\times 10^{3}\end{array}$ & $\begin{array}{l}\text { time } \\
\text { (hrs) } \\
\text { [aborted] }\end{array}$ & $\begin{array}{l}\# \text { cnfls } \\
\times 10^{3}\end{array}$ & $\begin{array}{l}\text { time } \\
\text { (hrs) }\end{array}$ & $\begin{array}{l}\# \text { cnfls } \\
\times 10^{3}\end{array}$ & $\begin{array}{l}\text { time } \\
\text { (hrs) }\end{array}$ \\
\hline Sat. & 28 & $>488$ & $>65[4]$ & $>2,204$ & $>40[2]$ & 3,406 & 16.4 & 756 & 4.5 \\
\hline Unsat. & 51 & $>1,603$ & $>87[1]$ & $>1,772$ & $>27[1]$ & 1,269 & 4.0 & 1,448 & 9.5 \\
\hline Total & 79 & $>2,091$ & $>152[5]$ & $>3,976$ & $>67[3]$ & 4,675 & 20.4 & 2,204 & 14.0 \\
\hline
\end{tabular}

\subsection{Can FI be simulated by a random windowing of variables?}

In the previous section, we saw that in the number of backtracks, $F I$ is competitive with state-of-the-art SAT-solvers. One might think that this result can be attributed to the great robustness of decision making based on computing conflict activity of variables (or literals). It might be the case that even if a SAT-solver branches on a variable with a reasonably 


\section{E. GOLDBERG}

high activity score (instead of the most active variable), it still may get the same or similar results. Then even though $F I$ has a dramatically smaller pool of variables to select from, the set $\operatorname{Vars}(M(\boldsymbol{p}))$ may still contain variables with good activity scores.

Table 3. Comparison of FI and Minisat on a sample of large BMC formulas

\begin{tabular}{|l|l|l|l|l|l|l|l|l|l|l|}
\hline Name & \#vars & \#cla & \multicolumn{3}{l|}{ Minisat } & \multicolumn{2}{l|}{$F I$} \\
\cline { 5 - 13 } & $\times 10^{3}$ & $\begin{array}{l}\text { uses } \\
\times 10^{3}\end{array}$ & $\begin{array}{l}\text { \#decis. } \\
\times 10^{3}\end{array}$ & $\begin{array}{l}\text { \#imp. } \\
\times 10^{6}\end{array}$ & $\begin{array}{l}\text { \#cnfl. } \\
\times 10^{3}\end{array}$ & $\begin{array}{l}\text { time } \\
(\mathrm{s})\end{array}$ & $\begin{array}{l}\text { \#dec. } \\
\times 10^{3}\end{array}$ & $\begin{array}{l}\text { \#impl. } \\
\times 10^{6}\end{array}$ & $\begin{array}{l}\text { \#cnfl. } \\
\times 10^{3}\end{array}$ & $\begin{array}{l}\text { time } \\
(\mathrm{s})\end{array}$ \\
\hline always.100 & 249 & 758 & 379 & 168 & 21 & 211 & 83 & 109 & 16 & $\mathbf{1 5 7}$ \\
\hline mcbdm.40 & 273 & 773 & 231 & 50 & 6 & 84 & 59 & 19 & 9 & $\mathbf{2 8}$ \\
\hline cfet.22 & 613 & 1,808 & 1,763 & 98 & 14 & 385 & 368 & 23 & 31 & $\mathbf{5 8}$ \\
\hline ccc.16 & 317 & 1,081 & 163 & 187 & 22 & $\mathbf{1 5 0}$ & 44 & 375 & 21 & 1,559 \\
\hline *sched.30 & 974 & 2,653 & 3,805 & 834 & 23 & 937 & 245 & 643 & 26 & $\mathbf{7 3 7}$ \\
\hline *prop9.100 & 1,001 & 2,981 & 521 & 599 & 40 & $\mathbf{4 1 1}$ & 305 & 549 & 48 & 1,190 \\
\hline *iuf.200 & 2,083 & 6,443 & 134,305 & 3,891 & 593 & 9,873 & 57 & 24 & 6 & $\mathbf{5 9}$ \\
\hline *fft.2000 & 260 & 736 & 54,883 & 2,188 & 148 & 2,433 & 26 & 947 & 1 & $\mathbf{8}$ \\
\hline
\end{tabular}

Table 4. Comparison of Minisat and FI in terms of decision and implied assignments

\begin{tabular}{|l|l|l|l|l|}
\hline \multirow{3}{*}{ Category } & \multicolumn{2}{|l|}{ Minisat } & FI \\
\cline { 2 - 5 } & $\begin{array}{l}\text { \#decis. } \\
\times 10^{6}\end{array}$ & $\begin{array}{l}\text { \#impl. } \\
\times 10^{6}\end{array}$ & $\begin{array}{l}\text { \#decis. } \\
\times 10^{6}\end{array}$ & $\begin{array}{l}\text { \#impl. } \\
\times 10^{6}\end{array}$ \\
\hline Sat. & 91 & 10,616 & 10 & 5,979 \\
\hline Unsat. & 587 & 40,003 & 15 & 12,979 \\
\hline Total & 679 & 50,619 & 25 & 18,958 \\
\hline
\end{tabular}

To resolve this issue we ran some experiments on formulas of Table 2 (we observed similar results on other classes of benchmarks). The idea was to compare FI with a SATsolver whose only difference from $F I$ was in the way the set of branching variables was restricted. In $F I$, we pick the next variable to branch on out of $\operatorname{Vars}(M(\boldsymbol{p}))$ (i.e. the variables of the clauses falsified by the current complete assignment $\boldsymbol{p}$ ). We compared $F I$ with a SAT-solver that randomly chooses a window of $|\operatorname{Vars}(M(\boldsymbol{p}))|$ variables and then picks the most active literal among the window variables. If the good performance of $F I$ is due to great robustness of decision making based on variable/literal activity, then the performance of these two SAT-solvers should be close.

The results of experiments are shown in Tables 5 and 6. Both tables have identical structure and give data for unsatisfiable and satisfiable formulas. The second and third columns describe the number of variables and clauses (in thousands). The fourth and fifth columns give the number of conflicts and the average window size (in percent) for $F I$ computed over all decision assignments. For every decision, the window size was computed for the current set of variables whose values had not been fixed yet. For example, if, in 
the current formula, there are 100,000 of such variables and $|\operatorname{Vars}(M(\boldsymbol{p}))|=1000$, then the window size is 1\%. For the formula pa.25 (first formula of Table 5) the average window size (computed over all decision assignments) was $0.43 \%$.

The column before the last shows the results of the SAT-solver that used random windowing. For example, for the formula pa.25, for all decisions we used windows of the size $0.43 \%$ (the average size of $F I$ windows for this formula). For instance, if the current set of available branching variables was 100,000, then this SAT-solver would generate a random window $W$ of 430 variable and then picks the most active literal among the variables of $W$. For the formula pa.25, the SAT-solver with random windowing proved unsatisfiability after 374 backtracks (conflicts). The last column gives the result when the most active literal was selected among all available branching variables (i.e. the window size was 100\%).

The results of Table 5 and 6 show the advantage of windowing of $F I$ over random windowing. So one can not claim that good performance of $F I$ is due to robustness of decision making based on computing conflict activity of literals. Interestingly, the advantage of $F I$ windowing is greater for satisfiable formulas. Only in one case (fetch.30), the SAT-solver with random windowing had fewer backtracks before finding a satisfying assignment. For the rest of the formulas, FI found a satisfying assignment with a considerably smaller number of backtracks. The results of this subsection show that windows specified by complete assignments are "special". So these results can be considered as a strong argument in favor of proof encryption semantics.

Note that, in general, the SAT-solver choosing the most active literal out of all available variables, had fewer backtracks than FI. But as we saw in the previous subsection the number of decision and implied assignments (not shown here) made by such a SAT-solver may be much larger than for $F I$.

Table 5. Different choice of windows for unsatisfiable formulas

\begin{tabular}{|l|l|l|l|l|l|l|}
\hline \multirow{2}{*}{ Name } & $\begin{array}{l}\text { \#Vars } \\
\times 10^{3}\end{array}$ & $\begin{array}{l}\text { \#Clauses } \\
\times 10^{3}\end{array}$ & \multicolumn{2}{l|}{$F I$} & \multicolumn{2}{l|}{ no complete assgn. } \\
\cline { 5 - 7 } & & & \#cnfl. & $\begin{array}{l}\text { window } \\
\text { size }(\%)\end{array}$ & $\begin{array}{l}\text { \#cnfl. } \\
\text { (window) }\end{array}$ & $\begin{array}{l}\text { \#cnfl } \\
\text { (all vars) }\end{array}$ \\
\hline pa.25 & 337 & 995 & 127 & 0.43 & 374 & 88 \\
\hline pa.50 & 727 & 2,146 & 1,436 & 2.02 & 46,590 & 480 \\
\hline mcbdm.20 & 127 & 360 & 157 & 2.03 & 549 & 163 \\
\hline mcbdm.40 & 273 & 773 & 2,019 & 1.22 & 12,280 & 8,700 \\
\hline cmt.100 & 63 & 178 & 194 & 0.03 & 9,753 & 469 \\
\hline cmt.200 & 127 & 358 & 403 & 0.01 & 27,529 & 1,715 \\
\hline always.20 & 61 & 186 & 158 & 1.28 & 238 & 244 \\
\hline always.40 & 136 & 415 & 2,955 & 2.89 & 4,763 & 1,994 \\
\hline pc_top.40 & 379 & 1,126 & 1,066 & 0.41 & 5,447 & 752 \\
\hline pc_top.50 & 480 & 1,424 & 3,609 & 0.52 & 11,786 & 3,747 \\
\hline lsu.10 & 89 & 239 & 3,253 & 1.01 & 7.015 & 1,024 \\
\hline stdata.10 & 110 & 302 & 2,764 & 1.65 & 7,901 & 725 \\
\hline
\end{tabular}


Table 6. Different choice of windows for satisfiable formulas

\begin{tabular}{|c|c|c|c|c|c|c|}
\hline \multirow{2}{*}{ Name } & \multirow{2}{*}{$\begin{array}{l}\text { \#Vars } \\
\times 10^{3}\end{array}$} & \multirow{2}{*}{$\begin{array}{l}\text { \#Clauses } \\
\times 10^{3}\end{array}$} & \multicolumn{2}{|l|}{$F I$} & \multicolumn{2}{|c|}{ no complete assgn. } \\
\hline & & & \#cnfl. & $\begin{array}{l}\text { window } \\
\text { size }(\%)\end{array}$ & $\begin{array}{l}\text { \#cnfl. } \\
\text { (window) }\end{array}$ & $\begin{array}{l}\text { \#cnfl } \\
\text { (all vars) }\end{array}$ \\
\hline sched.10 & 188 & 508 & 159 & 0.36 & 4,992 & 60 \\
\hline sched.20 & 384 & 1,044 & 1,367 & 0.51 & 21,038 & 389 \\
\hline byteen.10 & 69 & 184 & 1,007 & 1.25 & 7,093 & 1,566 \\
\hline byteen. 15 & 110 & 292 & 5,196 & 1.55 & 21,277 & 5,171 \\
\hline bar.10 & 371 & 1,102 & 1,157 & 0.08 & 56,594 & 36 \\
\hline gmtx.50 & 645 & 1,887 & 708 & 2.42 & 12,298 & 199 \\
\hline muls.20 & 307 & 944 & 647 & 0.20 & 594,669 & 57 \\
\hline fetch.30 & 168 & 501 & 325 & 2.29 & 19 & 168 \\
\hline write. 40 & 233 & 695 & 1,949 & 1.24 & 46,773 & 1,172 \\
\hline prop7.50 & 496 & 1,475 & 1,527 & 1.35 & 86,349 & 148 \\
\hline resp_grant.20 & 302 & 892 & 877 & 2.97 & 9,775 & 82 \\
\hline
\end{tabular}

Table 7. Formulas with unsatisfiable core (1 hour timeout)

\begin{tabular}{|l|l|l|l|}
\hline Names & $\begin{array}{l}\text { BerkMin561 } \\
\text { \#conflicts }\end{array}$ & $\begin{array}{l}\text { Minisat } \\
\text { \#conflicts }\end{array}$ & $\begin{array}{l}\text { FI } \\
\text { \#conflicts }\end{array}$ \\
\hline f10k10_1 & $>2,354,579$ & $>1,586,998$ & 756 \\
\hline f10k10_2 & $>2,530,886$ & $>1,509,013$ & 935 \\
\hline f10k10_3 & $>2,014,759$ & $>1,519,403$ & 908 \\
\hline f10k10 & 4 & 6 & 1,209 \\
\hline f3k50_1 & $>6,542,130$ & $>3,188,959$ & 48 \\
\hline f3k50_2 & $>6,538,252$ & $>4,300,869$ & 18,127 \\
\hline f3k50_3 & $>6,479,246$ & 79 & 18,890 \\
\hline f3k50 & 74 & 71 & 15,447 \\
\hline
\end{tabular}

\subsection{Finding unsatisfiable subformulas}

In this subsection, we consider the performance of BerkMin, Minisat and FI on 8 artificial formulas with small unsatisfiable subformulas. The results are shown in Table 7 . Formulas $f 10 k 10 \_i$ and $f 3 k 50 \_i, i=1,2,3$ were obtained from formulas $f 10 k 10$ and $f 3 k 50$ by random permutation of variables. The formula $f 10 k 10$ was obtained by adding to a hard random formula $F_{1}$ of 10,000 variables the clauses of a random unsatisfiable formula $F_{2}$ of 10 new variables. The formula $f 3 k 50$ was obtained in the same way as $f 10 k 10$. The only difference was that formula $F_{1}$ had 3,000 variables and $F_{2}$ had 50 variables. (Note that we got results similar to those of Table 7 even when variables of $F_{1}$ and $F_{2}$ had a "weak" overlap.) Timeout limit was set to 1 hour. 
As we mentioned in Subsection 4.1, one of the advantages of operating on complete assignments is easy identification of unsatisfiable subformulas. For a formula $F_{1} \wedge F_{2}$ of Table 7, no matter how FI picks a complete assignment $\boldsymbol{p}$, the set $M(\boldsymbol{p})$ will contain a clause of the unsatisfiable subformula $F_{2}$. In this experiment, we modified the first decisionmaking heuristic of FI (described in Subsection 2.4) as follows. For every clause $C$ of the formula, its activity was maintained. This activity was computed as the number of conflicts in which $C$ was involved. Every 100 decisions $F I$ picked the clause $C^{\prime}$ of $M(\boldsymbol{p})$ with the lowest activity and fixed an assignment to a variable of $C^{\prime}$. This way we made $F I$ to look for unsatisfiable subformulas (because the modification above forced $F I$ to fix an assignment to a variable of $F_{2}$ once in a while). Note that this modification does not change FI's performance on other formulas much because in $99 \%$ cases FI makes a regular decision. As one can see from Table 7, BerkMin and Minisat easily solved 2 and 3 formulas respectively, but for the rest of the formulas they got stuck in the hard subformula $F_{1}$. On the other hand, FI easily solved all 8 formulas.

\section{Conclusions and future directions}

In this paper, we introduce a proof encryption semantics whose main idea is to treat a set of complete assignments as an "encryption" of a resolution proof. We believe that this semantics better explains what resolution-based SAT-solvers with clause learning do. We describe a SAT-solver FI inspired by the proof encryption semantics. FI can be viewed as a regular DPLL-like SAT-solver that maintains a complete assignment to reduce the choice of variables to branch on. Experiments show that for large industrial formulas even our primitive implementation of $F I$ is competitive with Minisat and BerkMin. Experiments also give a strong evidence in favor of proof encryption semantics. Namely they show that FI can not be efficiently simulated by random windowing of branching variables.

\section{References}

[1] L. Bachmair and H. Ganzinger. Resolution theorem proving in A.Robinson, A.Voronkov editors, The Handbook of Automated Reasoning, chap. 2, vol. 1, pp. 19-99. Elsevier Science Pub., 2001.

[2] P. Beame, H. A. Kautz, and A. Sabharwal. Towards Understanding and Harnessing the Potential of Clause Learning. JAIR 2004, number 22, pp.319-351.

[3] M. Davis, G. Logemann, and D.Loveland. A Machine program for theorem proving. Communications of the ACM, 1962, vol. 5, pp. 394-397.

[4] N. Eén. and N. Sörensson. An extensible SAT-solver. Proceedings of SAT-2003 in LNCS 2919, pp.503-518.

[5] H. Fang and W. Ruml. Complete Local Search for Propositional Satisfiability. Proc. of $19^{\text {th }}$ National Conference on Artificial Intelligence, 2004, pp.161-166.

[6] E. Goldberg. Testing satisfiability of CNF formulas by computing a stable set of points. CADE-2002, pp. 161-180. 
[7] E. Goldberg. On bridging simulation and formal verification. VMCAI-2008, San Francisco, USA, LNCS 4905, pp.127-141.

[8] E. Goldberg and Y. Novikov. BerkMin: a Fast and Robust SAT-Solver. DATE-2002, pp. 142-149.

[9] E. Goldberg and Y. Novikov. Verification of proofs of unsatisfiability for CNF formulas. DATE-2003, pp. 886-891.

[10] C. P. Gomes, B. Selman, H. A. Kautz. Boosting combinational search through randomization. AAAI-1998, pp. 431-437.

[11] D. Habet, C. M. Li, L. Devendeville, and M. Vasquez. A hybrid approach for SAT. International Conference on Principles and Practice of Constraint Programming, 2002, pp. $172-184$.

[12] E. Hirsch, A. Kojevnikov. UnitWalk: A new SAT solver that uses local search guided by unit clause elimination. Annals of Math. and Artif. Intelligence 2005, vol. 43(1-4), pp.91-111.

[13] B. Mazure, L. Sais, and R. Gregoire. Boosting complete techniques thanks to local search methods. Annals of Math. and Artif. Intelligence 1998, vol. 22, pp. 319-331.

[14] M. Moskewicz, C. Madigan, Y. Zhao, L. Zhang, and S. Malik. Chaff: Engineering an Efficient SAT Solver. Design Automation Conference, 2001, pp. 530-535.

[15] S. Prestwich and I. Lynce. Local Search for Unsatisfiability. SAT-2006, LNCS 4121, pp. 283-296.

[16] B. Selman, H. Levesque, and D. Mitchell. A New Method for Solving Hard Satisfiability Problems. AAAI-92, pp. 440-446.

[17] B. Selman, H. A. Kautz, and B.Cohen. Noise strategies for improving local search. AAAI-94, Seattle, pp. 337-343.

[18] B. Selman, H. A. Kautz, and D. McAllester. Ten challenges in propositional reasoning and search. IJCAI-97, Nagoya, Aichi, Japan.

[19] J. P. Marques-Silva and K. Sakallah. GRASP: A Search Algorithm for Propositional Satisfiability. IEEE Transactions of Computers, 1999, vol 48, pp. 506-521.

[20] H. Zhang. SATO: An efficient propositional prover. Proceedings of the International Conference on Automated Deduction, 1997, pp. 272-275.

[21] L. Zhang, C. Madigan, M. Moskewicz, S. Malik. Efficient Conflict Driven Learning in a Boolean Satisfiability Solver, ICCAD-2001, San Jose, pp. 279-285.

[22] http://www.cadence.com/webforms/cbl_software/index.aspx 